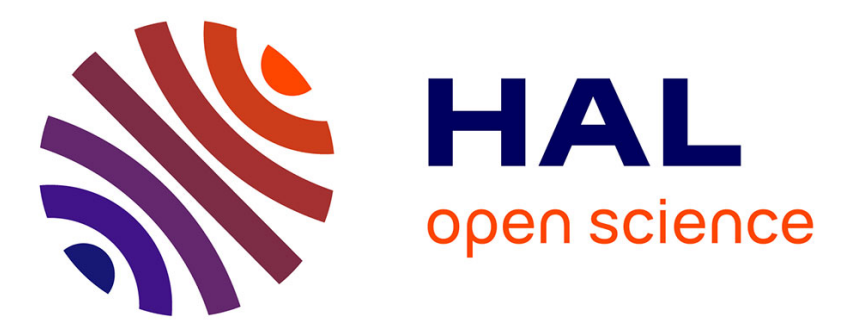

\title{
Infrared and Raman spectroscopic features of the self-interstitial defect in diamond from exact-exchange hybrid DFT calculations
}

\author{
Simone Salustro, Alessandro Erba, Claudio Zicovich-Wilson, Yves Noël, \\ Lorenzo Maschio, Roberto Dovesi
}

\section{To cite this version:}

Simone Salustro, Alessandro Erba, Claudio Zicovich-Wilson, Yves Noël, Lorenzo Maschio, et al.. Infrared and Raman spectroscopic features of the self-interstitial defect in diamond from exactexchange hybrid DFT calculations. Physical Chemistry Chemical Physics, 2016, 18, pp.21288-21295. 10.1039/C6CP02403C . hal-01404012

\section{HAL Id: hal-01404012 https://hal.science/hal-01404012}

Submitted on 6 Feb 2017

HAL is a multi-disciplinary open access archive for the deposit and dissemination of scientific research documents, whether they are published or not. The documents may come from teaching and research institutions in France or abroad, or from public or private research centers.
L'archive ouverte pluridisciplinaire HAL, est destinée au dépôt et à la diffusion de documents scientifiques de niveau recherche, publiés ou non, émanant des établissements d'enseignement et de recherche français ou étrangers, des laboratoires publics ou privés. 


\title{
Journal Name
}

\section{ARTICLE TYPE}

Cite this: DOI: 10.1039/xxxxxxxxxx

\section{Infrared and Raman Spectroscopic Features of the Self-Interstitial Defect in Diamond from Exact- Exchange Hybrid DFT Calculations}

\author{
Simone Salustro, ${ }^{a}$ Alessandro Erba, ${ }^{a}$ Claudio M. Zicovich-Wilson, ${ }^{b}$ Yves Nöel ${ }^{c}$ \\ Lorenzo Maschio ${ }^{a}$ and Roberto Dovesi ${ }^{a}$
}

Received Date

Accepted Date

DOI: 10.1039/xxxxxxxxxx

www.rsc.org/journalname

\begin{abstract}
Quantum-mechanical calculations are performed to investigate structural, electronic, and Infrared (IR) and Raman spectroscopic features of one of the most common radiation-induced defects in diamond: the "dumb-bell" $\langle 100\rangle$ split self-interstitial. A periodic super-cell approach is used in combination with all-electron basis sets and hybrid functionals of the density-functional-theory (DFT), which include a fraction of exact non-local exchange and are known to provide a correct description of the electronic spin localization at the defect, at variance with simpler formulations of the DFT. The effects of both defect concentration and spin state are explicitly addressed. Geometrical constraints are found to prevent the formation of a double bond between the two three-fold coordinated carbon atoms. On the contrary, two unpaired electrons are fully localized on each of the carbon atoms involved in the defect. The open-shell singlet state is slightly more stable than the triplet (the energy difference being just $30 \mathrm{meV}$, as the unpaired electrons occupy orthogonal orbitals) while the closed-shell solution is less stable by about $1.55 \mathrm{eV}$. The formation energy of the defect from pristine diamond is about $12 \mathrm{eV}$. The Raman spectrum presents only two peaks of low intensity at wave-numbers higher than the pristine diamond peak (characterized by normal modes extremely localized on the defect), whose positions strongly depend on defect concentration as they blue shift up to 1550 and $1927 \mathrm{~cm}^{-1}$ at infinite defect dilution. The first of these peaks, also IR active, is characterized by a very high IR intensity, and might then be related to the strong experimental feature of the IR spectrum occurring at $1570 \mathrm{~cm}^{-1}$. A second very intense IR peak appears at about $500 \mathrm{~cm}^{-1}$, which, despite being originated from a "wagging" motion of the self-interstitial defect, exhibits a more collective, less localized character.
\end{abstract}


Raman peak at $1332 \mathrm{~cm}^{-1}$, defective samples are characterized by several additional features, the most significant ones being observed at about 1450, 1490, 1630 and $1680 \mathrm{~cm}^{-1}$. 3,19 The spectral features at 1630 and $1680 \mathrm{~cm}^{-1}$ have been attributed to $\mathrm{sp}^{2}$ defects. In particular, the peak at $1630 \mathrm{~cm}^{-1}$ has been experimentally assigned to the "dumb-bell" split self-interstitial defect. $^{3}$ Despite this assignation was originally confirmed by molecular dynamics theoretical simulations using a Brenner semiempirical potential, ${ }^{13}$ a more recent $a b$ initio density-functionaltheory (DFT) simulation performed on finite clusters using a generalized-gradient functional could not confirm it. ${ }^{14}$

Many possible interstitial defects in diamond have been proposed in the literature in the last 20 years $(\langle 100\rangle,\langle 110\rangle$, bondcentered, platelets, di-[001] split interstitial, Humble di-[001] split interstitial), whose structural, electronic and energetic properties have been theoretically investigated in a series of papers by Briddon and coworkers; ${ }^{1,5,24}$ the most stable isolated interstitial defect was found to be the $\langle 100\rangle$ split self-interstitial one.

From a theoretical perspective, achieving a correct description of the electronic states of the defects within the main energy band-gap of the semiconductor is crucial, as well as describing the various possible spin couplings and the related role of symmetry. A non-trivial description of non-local long-ranged interactions is thus needed. These issues have been discussed in a seminal paper by Coulson and Kearsley about 60 years ago, using rather simple models. ${ }^{25}$ Since $a b$ initio techniques have become feasible, both the finite cluster and the periodic super-cell approaches have been used, ${ }^{24}$ mostly as combined with the simplest formulation of the DFT (the local-density approximation, LDA), which is known to poorly describe the exchange interactions of well-localized unpaired electrons and to largely underestimate energy band-gaps, where defect states are located.

Since theoretical simulations of IR and Raman spectra of extended solids have become feasible at the quantum-mechanical level of theory, computational spectroscopy has become an effective complementary tool in the interpretation of experimental spectra, and an essential mean to the atomistic characterization of their features. ${ }^{26-33}$ In the present study, $a b$ initio calculations are performed to investigate the electronic and spectroscopic properties of the self-interstitial $\langle 100\rangle$ defect in diamond, by use of a periodic super-cell approach and of the hybrid B3LYP functional, which contains $20 \%$ of non-local exact exchange (an essential prerequisite to the correct description of the electronic spin localization at the defect), ${ }^{34-39}$ as implemented in the CRYSTAL14 program. ${ }^{33}$ The effects of different defect concentrations and spin states are explicitly explored. The same computational procedure has recently been adopted in the theoretical characterization of electronic and Raman spectroscopic features of the neutral vacancy in diamond, which has allowed to rule out the previous assignation of the spectral features at 1450 and $1490 \mathrm{~cm}^{-1}$ as due to the vacancy. 40,41

\section{Computational Models and Details}

All spin-polarized DFT calculations have been performed by use of the B3LYP global hybrid functional, ${ }^{42,43}$ as implemented in the CRYSTAL14 program. ${ }^{33}$ Some of the preliminary calculations on electronic and energetic features of the defects have also been repeated with the unrestricted Hartree-Fock (HF) method and with other DFT functionals ranging from pure ( $\mathrm{LDA}^{44}$ and $\mathrm{PBE}^{45}$ ), to global hybrid (PBE0 ${ }^{46}$ ), and range-separated hybrid (HSE06 ${ }^{47}$ ) ones. An all-electron basis set of Gaussian-type functions has been adopted (Pople's 6-21G), whose outermost exponent (0.228 Bohr $^{-2}$ ) of the most diffuse $s p$ shell has been reoptimized for bulk diamond. ${ }^{48}$ The defect formation energy has also been calculated with larger basis sets, such as 6-21G*, 6-31G and 6-31G*. 49 The truncation of the Coulomb and exchange infinite lattice series is controlled by five thresholds $T_{i}$, which have been set to $8\left(T_{1}-T_{4}\right)$ and $16\left(\mathrm{~T}_{5}\right)$. The convergence threshold on energy for the selfconsistent-field (SCF) procedure has been set to $10^{-8}$ hartree for structural optimizations and to $10^{-10}$ hartree for vibration frequency calculations.

Different structural models and spin-states of the selfinterstitial defect of diamond are considered, which correspond to different point-symmetries: the $\langle 100\rangle$ split interstitial in the singlet state to $C_{2 v}$, the $\langle 100\rangle$ split interstitial in the triplet state to $D_{2 d}$, and the bond-centered (BC) interstitial to $C_{3 v}$. A periodic super-cell approach is used to simulate different defect concentrations. Super-cells containing 33, 65 and 129 atoms are considered (to be referred to in the following as $S_{n}$, with $n=33,65$ or 129), which correspond to defect densities of $5.53 \cdot 10^{21} \mathrm{~cm}^{-3}, 2.76 \cdot 10^{21}$ $\mathrm{cm}^{-3}$ and $1.38 \cdot 10^{21} \mathrm{~cm}^{-3}$, respectively. Reciprocal space has been sampled using a regular sub-lattice with a shrinking factor of 8 for $S_{33}$ and $S_{65}$ and of 4 for $S_{129}$, which correspond to a sampling over 59, 65 and $13 \mathrm{k}$-points in the irreducible part of the Brillouin zone for the self-interstitial defect of $D_{2 d}$ point-symmetry.

Harmonic phonon frequencies (i.e. wave-numbers), $\omega_{p}$, at the $\Gamma$ point (i.e. at the center of the first Brillouin zone in reciprocal space) are obtained from the diagonalization of the massweighted Hessian matrix of the second energy derivatives with respect to atomic displacements $u:^{29,50-53}$

$$
W_{a i, b j}^{\Gamma}=\frac{H_{a i, b j}^{\mathbf{0}}}{\sqrt{M_{a} M_{b}}} \quad \text { with } \quad H_{a i, b j}^{\mathbf{0}}=\left(\frac{\partial^{2} E}{\partial u_{a i}^{\mathbf{0}} \partial u_{b j}^{\mathbf{0}}}\right),
$$

where atoms $a$ and $b$ (with atomic masses $M_{a}$ and $M_{b}$ ) in the reference cell, $\mathbf{0}$, are displaced along the $i$-th and $j$-th Cartesian directions, respectively. The Raman intensity of the Stokes line of a phonon mode $Q_{p}$, characterized by a frequency $\omega_{p}$, active due to the $\alpha_{i i^{\prime}}$ component of the polarizability tensor $\alpha$, is given by:

$$
I_{i i^{\prime}}^{p} \propto\left(\frac{\partial \alpha_{i i^{\prime}}}{\partial Q_{p}}\right)^{2} .
$$

The relative Raman intensities of the peaks are computed analytically by exploiting a scheme, recently implemented in the CRYsTAL14 program, ${ }^{26,27}$ which constitutes an extension of the analytical calculation of IR intensities. ${ }^{27,28}$ Both schemes are based on the solutions of first- and second-order Coupled-PerturbedHartree-Fock/Kohn-Sham (CPHF/KS) equations. ${ }^{54,55}$ The Raman spectrum is then computed by considering the transverse-optical (TO) modes and by adopting a pseudo-Voigt functional form: a linear combination of a Lorentzian and a Gaussian curve with full width at half maximum of $8 \mathrm{~cm}^{-1}$. Raman intensities are nor- 

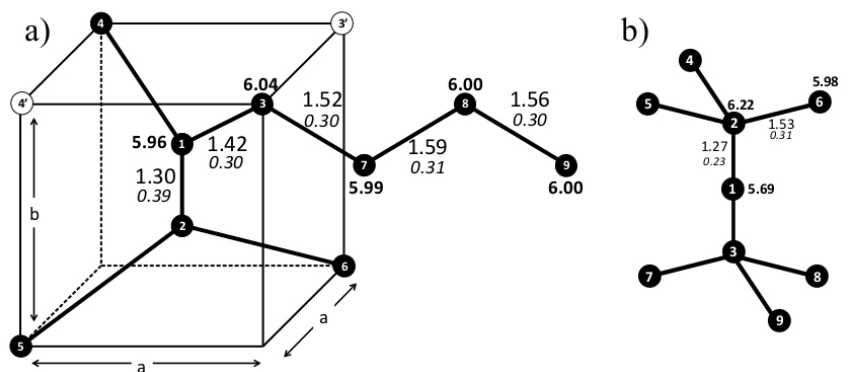

Fig. 1 Schematic representation of a) the $\langle 100\rangle$ split self-interstitial in the singlet state and $b$ ) the bond-centered interstitial in the triplet state in diamond. Selected values of bond lengths (in Å, large), Mulliken charges (in $|e|$, bold) and bond populations (in $|e|$, italic) are also reported as computed with the B3LYP hybrid functional for the largest super-cell $\left(S_{129}\right)$. The tetragonal symmetry of the $\langle 100\rangle$ defective diamond lattice is also highlighted in panel a) where $a=1.89 \AA$ and $b=2.28 \AA$.

malized so that the highest value is conventionally set to 1000 a.u. Integrated intensities for IR absorption $\mathscr{I}_{p}$ are computed for each mode $p$ by means of the mass-weighted effective-mode Born-charge vector $\vec{Z}_{p} 56,57$ evaluated through a CPHF/KS approach: 27,28

$$
\mathscr{I}_{p} \propto\left|\vec{Z}_{p}\right|^{2}
$$

The DFT exchange-correlation contribution to the Fock matrix has been evaluated by numerical integration over the unit cell volume. Radial and angular points for the integration grid are generated through Gauss-Legendre radial quadrature and Lebedev two-dimensional angular point distributions. The default pruned grid with 75 radial and 974 angular points has been used. With such a dense grid, the first-order Raman peak of pristine diamond is found at $1317 \mathrm{~cm}^{-1}$, at variance with the $1333 \mathrm{~cm}^{-1}$ position reported in the previous investigation on the neutral vacancy in diamond, where a somehow poorer grid was used (55 radial points and 434 angular points). ${ }^{40}$

\section{Results and Discussion}

\subsection{Structural, Electronic and Energetic Properties}

As anticipated in the Introduction, several possible self-interstitial defects have been proposed for diamond, 1,5,14 among which the $\langle 100\rangle$ split self-interstitial is indicated to be the most stable one. ${ }^{1}$ In the present study, we investigate the $\langle 100\rangle$ defect (in different spin states), as well as its possible counterpart where a doublebond is formed, and the bond-centered (BC) one. A schematic representation of the structural features of the $\langle 100\rangle$ and $B C$ interstitials is given in Figure 1, where some structural and electronic indices are also reported, to be discussed below. Let us start from a detailed investigation of structural, electronic and energetic properties of the $\langle 100\rangle$ split self-interstitial.

Three different electronic states are considered for the $\langle 100\rangle$ defect: a closed-shell solution, in which spin-up and -down electrons are forced to occupy the same defective crystalline orbitals (DCO), and the two open-shell solutions, in which spin-up and spin-down electrons are allowed to occupy different DCOs, which correspond to the singlet $S_{z}=0$ and triplet $S_{z}=1$ states. In Table
Table 1 Energy differences (in mHartree) of the triplet $S_{z}=1$ spin state, $\Delta E^{01}=\left|E_{0}\right|-\left|E_{1}\right|$, and the closed-shell state, $\Delta E^{0 C}=\left|E_{0}\right|-\left|E_{C}\right|$, with respect to the singlet state as computed with the B3LYP hybrid functional are reported as a function of defect concentration. For the most stable singlet spin state, the $d_{12}$ distance between atoms $\mathrm{C}_{1}$ and $\mathrm{C}_{2}$, and the $d_{13}$ distance between atoms $\mathrm{C}_{1}$ and $\mathrm{C}_{3}$ (in $\AA$ ) are reported.

\begin{tabular}{lcccc}
\hline \hline & $\Delta \mathrm{E}^{01}$ & $\Delta \mathrm{E}^{0 C}$ & $d_{12}$ & $d_{13}$ \\
& & & & \\
$S_{33}$ & 1.0 & 57.6 & 1.321 & 1.434 \\
$S_{65}$ & 1.0 & 57.4 & 1.308 & 1.427 \\
$S_{129}$ & 1.0 & 57.7 & 1.303 & 1.421 \\
& & & & \\
\hline \hline
\end{tabular}

1 , we report the computed energy of the singlet state for three different defect concentrations (corresponding to the $S_{33}, S_{65}$ and $S_{129}$ super-cells), as well as the energy differences of the other two electronic states with respect to $S_{z}=0$. Some considerations: i) the two open-shell states are much more stable (by about 57 mHartree) than the closed-shell solution; ii) the singlet state is found to be slightly more stable than the triplet one, with an energy difference of about $1 \mathrm{mHartree}$ (i.e. $0.03 \mathrm{eV}$ ) thus indicating that the two unpaired electrons could either be kept sufficiently far apart from each other or occupy rather localized DCOs oriented on orthogonal planes, so as to make Pauli repulsion small (as clearly emerges from the spin-density maps reported in Figure S1 of the Supplementary Information); iii) the relative stabilities of the three electronic states are found to be almost independent of defect concentration. The higher stability of the singlet spin state seems to be in agreement with experimental evidences. ${ }^{2}$ In this respect, in order to check whether or not the computed higher stability of the singlet state could be an artifact of the specific adopted functional of the DFT, we have investigated it also by using several different methods (LDA, GGA, global and rangeseparated hybrid DFT functionals, and the HF method). The computed energy differences are reported in Table S1 of the Supplementary Information. All adopted methods describe a more stable singlet state (by about 1 mHartree for B3LYP, PBE0, HSE06 and PBE, 0.2 mHartree for LDA and 4 mHartree for HF).

As anticipated above, in Figure 1, we report selected values of structural and electronic quantities, which can be useful to describe the perturbation to the diamond lattice represented by the self-interstitial defect. For pristine diamond, the computed equilibrium bond length is of $1.56 \AA$, the covalent bond population is of $0.30|e|$ and, obviously, the electronic charge on each carbon atom is of $6.00|e|$. The bond length $d_{12}$ of the two central atoms of the $\langle 100\rangle$ defect is much shorter $(1.30 \AA$ ), with a corresponding higher bond population of $0.39|e|$ and a slightly lower electronic charge of $5.96|e|$. The spin density is found to be localized on the two central atoms as well, with spin moments $\mu= \pm 1.001$ $|e|$, which propagate (with sign alternation) in a rather damped way to first-neighbors, where $\mu=\mp 0.123|e|$ (almost one order of magnitude lower than that at the central atoms). It is seen that this perturbation propagates up to the fourth neighbors, where 

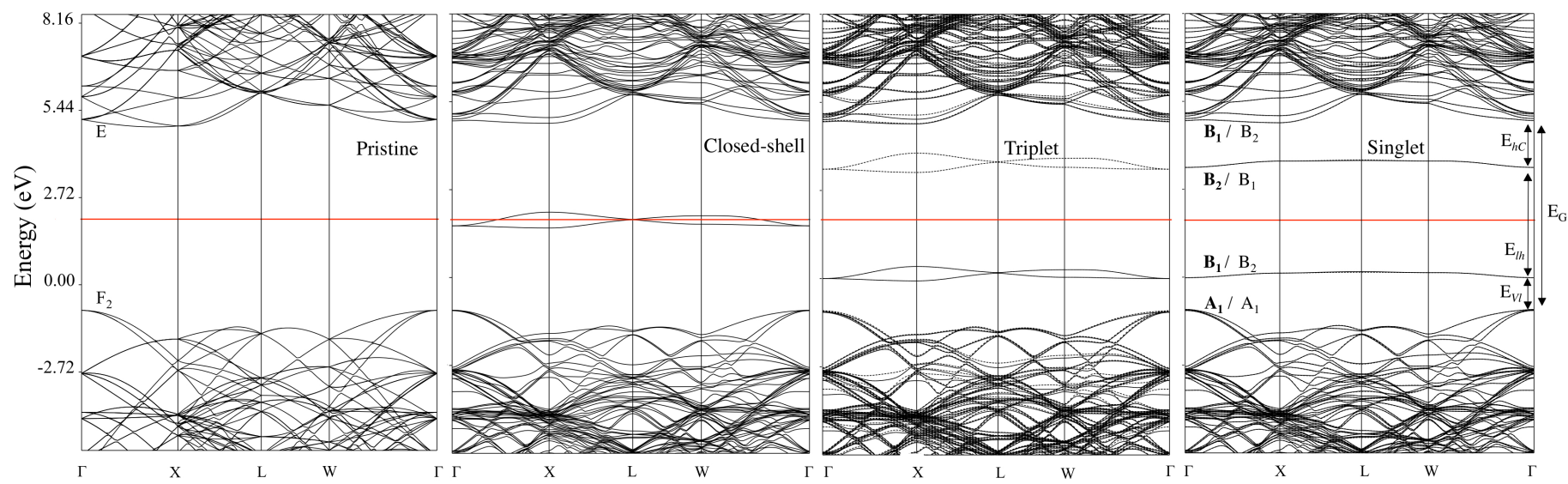

Fig. 2 (color online) Electronic band structure of pristine diamond and of the three spin states (closed-shell, triplet and singlet) of the $\langle 100\rangle$ split self-interstitial defect in the $S_{129}$ super-cell. Selected symmetry labels are given as determined at the $\Gamma$ point (in bold for spin-up and normal for spin-down electrons). The horizontal red line marks the Fermi energy. Continuous and dashed lines correspond to spin-up and spin-down bands, respectively.

the structural and electronic features of pristine diamond are fully recovered (see carbon atoms 8 and 9 in Figure 1).

Let us now discuss the formation energy $E_{f}$ of the $\langle 100\rangle$ split self-interstitial defect, which can be defined as ${ }^{58-60} E_{f}=E_{P}-$ $E_{D}-E_{A}$, where $E_{P}$ and $E_{D}$ are the total energies of pristine and defective diamond super-cells, and $E_{A}$ is the energy per atom in bulk pristine diamond. This formation energy is reported in Table 2 as computed with different theoretical methods (HF and several DFT functionals belonging to different rungs of "Jacob's ladder") and for different defect concentrations (i.e. with the three supercells: $S_{33}, S_{65}$ and $S_{129}$ ) for the most stable singlet spin state. For the B3LYP global hybrid functional, the effect on the computed formation energy of using richer basis sets is also documented. Some considerations: i) for a given defect concentration, the values of $E_{f}$ obtained with different methodologies are very consistent (despite the rather different descriptions they provide of spin localization); ii) the formation energy increases as the defect concentration decreases, passing from about $10.5 \mathrm{eV}$ for $S_{33}$, to about $11.5 \mathrm{eV}$ for $S_{65}$ and to $11.8 \mathrm{eV}$ for the lowest considered concentration $\left(S_{129}\right)$; iii) the computed formation energy of the defect is found to be very stable with respect to the enrichment of the adopted variational basis set, with a maximum difference of about $3 \%$. As a further check of the reliability of the computed formation energy, the effect of the inclusion of zero-point thermal effects has also been taken into account and has been found to be relatively small and to increase the defect formation energy by about $0.3 \mathrm{eV}$ for all methods.

In Figure 2, we report the electronic band structure of the $\langle 100\rangle$ split self-interstitial defect at low defect concentration (i.e. in the $S_{129}$ super-cell) in its three different spin states (closed-shell, singlet and triplet), as compared to that of pristine diamond (left panel). The Fermi energy is marked by a red horizontal line. If pristine diamond is described as an insulator with a band gap of $5.85 \mathrm{eV}$, the presence of the self-interstitial defect originates some localized electronic states within the gap. In the closed-shell solution, the double-occupancy with two electrons of the two nearly degenerate bands induces a metallic character to the so- lution. The singlet and triplet band structures correspond to nonconducting solutions and show minor differences between each other, thus confirming the small energy difference between the two states $(0.03 \mathrm{eV}$, as discussed above). Let us briefly illustrate the main features of the most stable (singlet) state: i) spin-up and spin-down COs are nearly degenerate at all k-points; ii) the main energy gap of pristine diamond basically remains unchanged and just splits into three sub-intervals, which are marked to the right of the singlet panel (about $1 \mathrm{eV}$ from the HOCO to the occupied defect band $E_{V l}$, about $3.4 \mathrm{eV}$ between the occupied and empty defect bands $E_{l h}$, and about $1.5 \mathrm{eV}$ from the empty defect band to the LUCO). The main features of such a band structure show just little dependence on defect concentration, as documented in Figure S2 of the Supplementary Information, the most significant effect being a reduced dispersion of the defect bands as the defect concentration decreases.

In order to evaluate whether or not a local structural distortion in the vicinity of the $\langle 100\rangle$ split self-interstitial defect (reducing the $C_{2 v}$ point-symmetry) could promote the formation of a

Table 2 Formation energies (in eV) of the $\langle 100\rangle$ split self-interstitial defect in diamond (singlet spin state) for different defect concentrations (i.e. for different super-cells: $S_{33}, S_{65}$ and $S_{129}$ ), as computed with several theoretical methods. For the B3LYP functional, the effect of using a richer basis set is also documented.

\begin{tabular}{llccc}
\hline \hline Method & Basis Set & $\mathrm{E}_{f}^{33}$ & $\mathrm{E}_{f}^{65}$ & $\mathrm{E}_{f}^{129}$ \\
& & & & \\
HF & $6-21$ & 10.75 & 11.77 & 12.01 \\
HSE06 & $6-21$ & 10.69 & 11.64 & 11.87 \\
PBE0 & $6-21$ & 10.74 & 11.64 & 11.87 \\
& $6-21$ & 10.45 & 11.37 & 11.62 \\
B3LYP & $6-21^{*}$ & 10.23 & 11.15 & 11.28 \\
& $6-31$ & 10.60 & 11.52 & 11.64 \\
PBE & $6-31^{*}$ & 10.48 & 11.40 & 11.57 \\
LDA & $6-21$ & 10.21 & 11.07 & 11.29 \\
& $6-21$ & 10.80 & 11.67 & 11.89 \\
\hline \hline
\end{tabular}


stabilizing double-bond between the two central atoms, we have performed a series of calculations where their first neighbors are rigidly rotated by a torsional angle up to planarity of the fragment involving atoms from $C_{1}$ to $C_{6}$ (see panel a of Figure 1). Both a cluster and a periodic model have been considered. While a detailed discussion of these calculations is given in the Supplementary Information, here let us summarize the main conclusions: i)

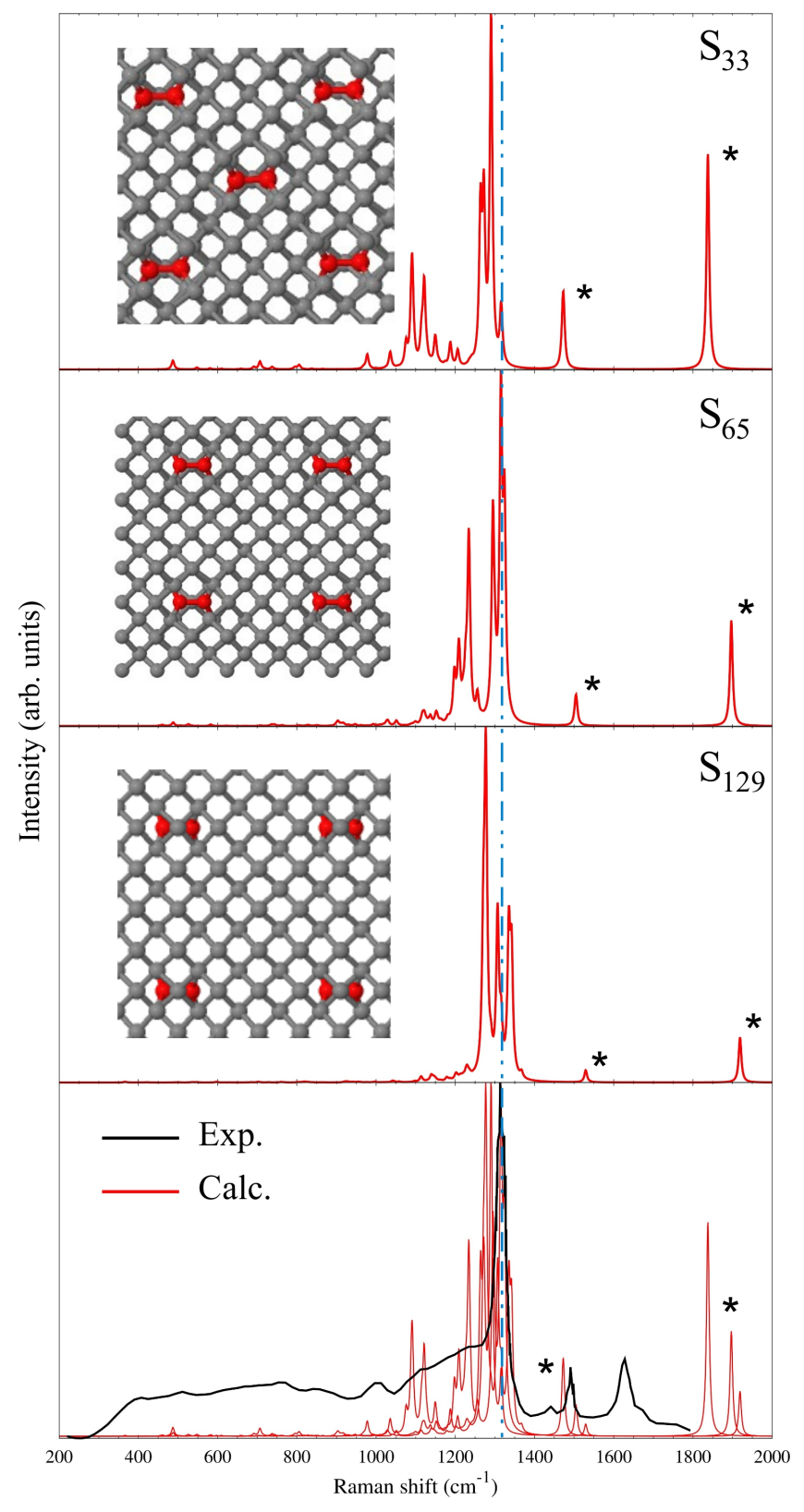

Fig. 3 (color online) Raman spectrum of the $\langle 100\rangle$ split self-interstitial defect in diamond, as computed at three defect concentrations (with the $S_{33}, S_{65}$ and $S_{129}$ super-cells). Insets of the three top panels show the structure of the defective system (with the two central atoms of the split interstitial defect in red). The intensity of the peaks marked with an asterisk are enhanced by a factor of 15 . The bottom panel reports a comparison between the experimental Raman spectrum of defective diamond ${ }^{19}$ (black) and computed spectra at different concentrations (red). The vertical dashed blue line marks the position of the first-order Raman peak in pristine diamond. the $C_{2 v}$ configuration given in Figure 1 (with no double-bond between the central atoms) is by far the most stable one with respect to lower-symmetry distorted configurations characterized by the formation of a partial to total double-bond; ii) a rigid rotation of first-neighbors of at least $30^{\circ}$ would be required to allow for the formation of a double-bond, which, however, would imply a much larger energy loss for the structural distortion with respect to the energy gain due to the double-bond formation.

The bond-centered interstitial defect (graphically represented in panel b of Figure 1) is here found to be less stable than the $\langle 100\rangle$ split self-interstitial one by about $2 \mathrm{eV}\left(2.1 \mathrm{eV}\right.$ for $S_{33}, 2.3 \mathrm{eV}$ for $S_{65}$ and $2.4 \mathrm{eV}$ for $S_{129}$ ). The origin of this large instability can be traced-back to the incapability of the central carbon atom to share two out of its four valence electrons, which remain unpaired and localized on it. This instability is reflected on a shorter $C_{1}-C_{2}$ bond length of $1.27 \AA$ (compared to $1.30 \AA$ in the $\langle 100\rangle$ defect) and a smaller bond population of $0.23|e|$ (compared to $0.39|e|$ in the $\langle 100\rangle$ defect). A relatively large charge transfer occurs from $C_{1}$ to $C_{2}$ as a consequence of the strong electron-electron repulsion.

\subsection{Raman Spectrum}

While it is generally rather difficult to experimentally assign given spectral features to specific structural defects (because of the large number of factors they might depend on, such as kind of defect, concentration, local distribution, etc.), this is not the case from a simulation point of view. With a computational approach indeed one has full control over the kind of defect to be investigated and the desired concentration.

We have computed the Raman vibrational spectrum (peak positions and intensities) of pristine and defective diamond by use of the fully-analytical scheme introduced in Section 2. The Raman spectrum of the $\langle 100\rangle$ split self-interstitial defect has been computed for the most stable singlet spin state and at three defect concentrations (with the $S_{33}, S_{65}$ and $S_{129}$ super-cells). These three spectra are reported in the first three top panels of Figure 3 , where the corresponding insets show the structure of the three models. The position of the first-order Raman peak of pristine diamond is marked by the vertical dashed blue line. The bottom panel of the figure reports a comparison between the experimental Raman spectrum of defective diamond measured up to 1800 $\mathrm{cm}^{-1}$ by Prawer et al. ${ }^{19}$ and present computed spectra at different defect concentrations.

At variance with what has been shown in the case of the neutral vacancy defect in diamond (where no defect-specific peaks are found above the first-order Raman peak of pristine diamond), ${ }^{40}$ the $\langle 100\rangle$ split self-interstitial defect produces a couple of peaks above the pristine diamond peak: one at about $1500 \mathrm{~cm}^{-1}$ and one at about $1900 \mathrm{~cm}^{-1}$. Some considerations on these two peaks: i) the peak position strongly depends on defect concentration, with a blue-shift as defect concentration decreases (the first peak passes from 1472 to 1504 to $1529 \mathrm{~cm}^{-1}$ and the second from 1838 to 1897 to $1921 \mathrm{~cm}^{-1}$ within the $S_{33}, S_{65}$ and $S_{129}$ super-cells, respectively); ii) the peak position changes linearly with the inverse of the number of atoms in the cell, which allows to extrapolate to infinite defect dilution (1550 and $1927 \mathrm{~cm}^{-1}$ ); 
iii) the intensity of the two peaks is relatively low (in the figure it has been magnified by a factor of 15); iv) the computed peak at about $1500 \mathrm{~cm}^{-1}$ is located in a region of the spectrum where an experimental peak has also been measured (at $1490 \mathrm{~cm}^{-1}$ ), and is likely to contribute to the experimental feature, even though, given its low intensity, other defects could contribute too; v) the second peak at about $1900 \mathrm{~cm}^{-1}$ is more intense and is found in a region where the experimental spectrum was not reported.

In order to explore whether or not the particular spin state of the self-interstitial defect could significantly affect the Raman spectrum in terms of peak positions, we have computed the vibrational spectrum of the defect both in its singlet and triplet state: differences in peak positions are found to be always smaller than $8 \mathrm{~cm}^{-1}$ (see Supplementary Information).

As anticipated in the Introduction, an assignation of the measured peak at about $1630 \mathrm{~cm}^{-1}$ to the "dumb-bell" split selfinterstitial defect was experimentally proposed ${ }^{3}$ and supported by molecular dynamics theoretical simulations using a Brenner semi-empirical potential. ${ }^{13}$ In line with a more recent $a b$ initio density-functional-theory (DFT) study, ${ }^{14}$ our present investigation rules out such an assignation as no Raman active modes are found in that spectral region.

\subsection{Infrared Spectrum}

Pristine diamond does not show any IR spectral feature because of its symmetry. On the contrary, irradiation-damaged diamond is characterized by a very intense feature in the IR experimental spectrum, at about $1570 \mathrm{~cm}^{-1}$, which has been tentatively assigned to the self-interstitial defect. ${ }^{2}$ In our previous study on IR and Raman spectra of the neutral vacancy in diamond, such a defect was found not to produce any intense features in the IR spectrum. This is clearly not the case for the $\langle 100\rangle$ split self-interstitial defect, which is indeed characterized by two intense IR peaks, at about 500 and $1550 \mathrm{~cm}^{-1}$ (the latter being also Raman active).

Figure 4 reports the computed IR spectrum of the $\langle 100\rangle$ split self-interstitial defect in diamond at three different defect concentrations (with the $S_{33}, S_{65}$ and $S_{129}$ super-cells), in the three top panels. The bottom panel reports a superposition of the three spectra, where the position of the experimentally measured IR peak is marked by a vertical dashed black line. As an eye-guide in the comparison with Figure 3, a vertical blue dashed line is also reported, which marks the position of the first-order Raman peak. Some considerations about the peak above $1500 \mathrm{~cm}^{-1}$ : i) while it has a very low Raman intensity (see again Figure 3), it constitutes a strong IR feature; ii) interestingly, its intensity increases as the defect concentration decreases; iii) as already discussed for the Raman spectrum, its position blue-shifts as the defect becomes more diluted, with a peak position at infinite dilution which is extrapolated to be at $1550 \mathrm{~cm}^{-1}$ (i.e. rather close to the experimentally measured feature at $1570 \mathrm{~cm}^{-1}$ ), thus confirming the tentative assignation of this peak to the self-interstitial defect. To further corroborate this assignment, we shall analyze below the corresponding vibrational normal mode.

\subsection{Normal Mode Analysis}

One of the great advantages of computational vibration spectroscopy is that, upon diagonalization of the mass-weighted Hessian matrix in Eq. (1), along with the eigenvalues (i.e. vibration frequencies related to peak positions) one gets also the corresponding eigenvectors, which can be analyzed to characterize the atomic motions involved in given spectral features. Normal modes of vibration can be analyzed either visually through graphical animations or via more quantitative schemes, such as the isotopic substitution of selected atoms. We have here followed both approaches to characterize the atomic motions proper of the most significant spectral features discussed in Sections 3.2 and 3.3. Graphical animations of the vibration modes of the $\langle 100\rangle$ split self-interstitial defect in diamond are available on-line, as obtained for the $S_{33}$ super-cell. ${ }^{61}$

The two normal modes corresponding to peaks above the first-

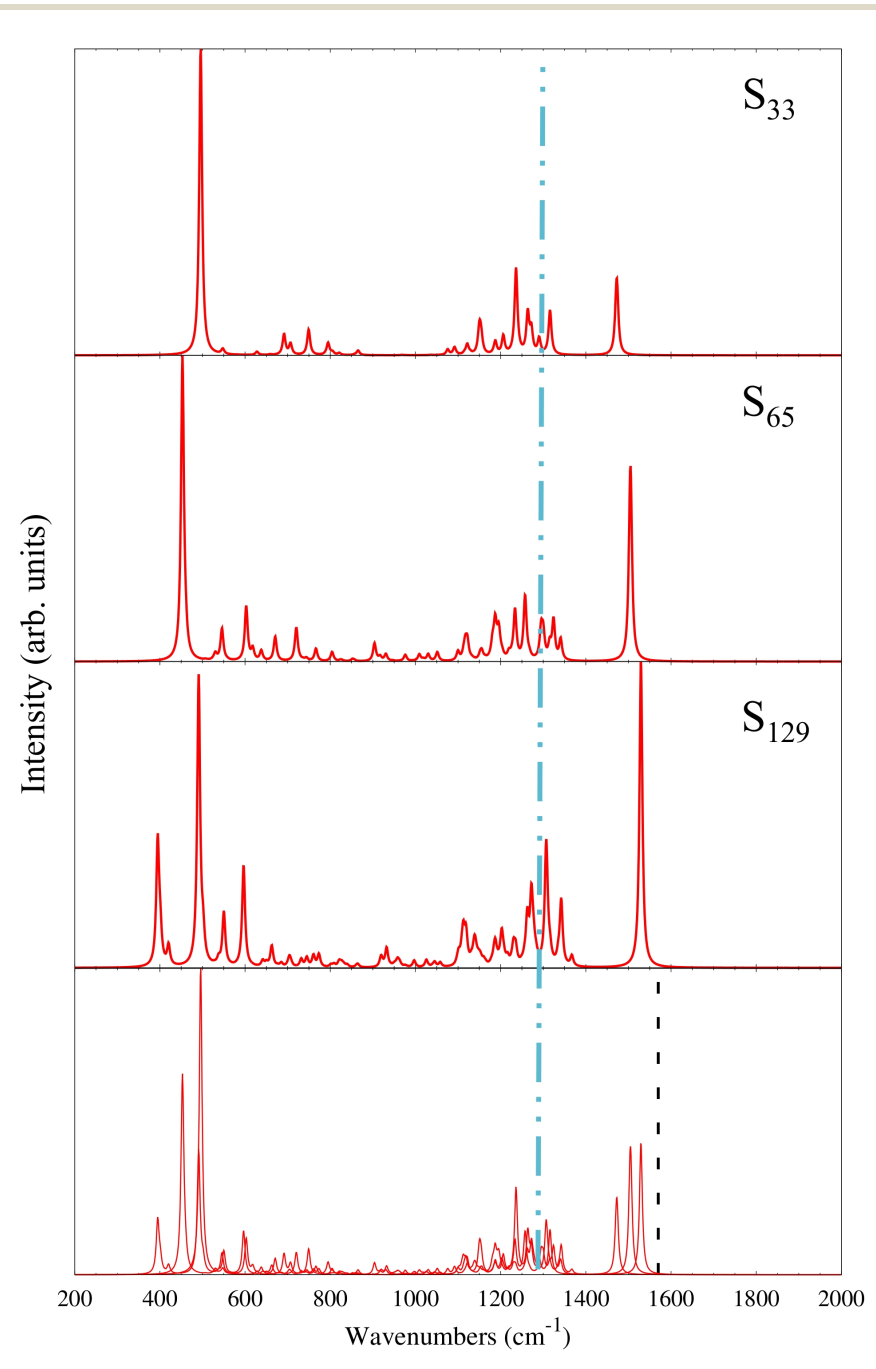

Fig. 4 (color online) IR spectrum of the $\langle 100\rangle$ split self-interstitial defect in diamond, as computed at three defect concentrations (with the $S_{33}$, $S_{65}$ and $S_{129}$ super-cells). The bottom panel reports a comparison between the experimental IR peak position of defective diamond ${ }^{2}$ (black dashed line) and computed spectra at different concentrations (red). The vertical dashed blue line marks the position of the first-order Raman peak in pristine diamond, as a guideline in the comparison with the Raman spectrum. 
order Raman one, at about $1900 \mathrm{~cm}^{-1}$ (Raman active) and 1500 $\mathrm{cm}^{-1}$ (both IR and Raman active), are extremely well-localized at the defect. The highest-frequency one is the $\mathrm{C}$-C bond stretching of the two central carbon atoms of the defect (atoms $C_{1}$ and $C_{2}$ in panel a of Figure 1). The other normal mode corresponds to the asymmetric stretching of the $C_{4}-C_{1}-C_{3}$ and $C_{5}-C_{2}-C_{6}$ bonds and is also extremely well-localized on the defect (just the two central atoms and their first-neighbors are involved). In order to confirm more quantitatively the localization of these modes on the defect, we have explored the effect of a localized isotopic substitution $\left({ }^{12} C \rightarrow{ }^{13} C\right)$ on the six carbon atoms $C_{1}$ to $C_{6}$. The effect of such a substitution is shown for both IR and Raman spectra in the Supplementary Information. The two peaks show a strong isotopic shift, which quantitatively corresponds to the ratio of the atomic masses $M_{{ }^{12} C} / M_{{ }^{13} C}$, as one would expect from Eq. (1), thus confirming the complete localization of the modes on the six central carbon atoms.

The intense IR spectral feature at about $500 \mathrm{~cm}^{-1}$ is due to a normal mode involving the "wagging" of the $C_{4}-C_{1}-C_{3}$ and $C_{5}-C_{2}-C_{6}$ sub-units, which then propagates to the other atoms of the covalently bonded carbon network, so as to result in a way less localized mode. The more collective character of such a mode is also reflected in a lower isotopic shift.

\section{Conclusions}

Electronic, energetic and vibrational spectroscopic features of the self-interstitial defect in diamond have been investigated via quantum-mechanical calculations performed by using an allelectron basis set and a hybrid functional of the DFT, which includes a fraction of exact non-local exchange, which is particularly well-suited to describe spin-polarized defective systems. Among other proposed configurations, the $\langle 100\rangle$ split selfinterstitial one if confirmed to be the most stable, in its singlet spin state, with a formation energy of about $12 \mathrm{eV}$.

At variance with the neutral vacancy, the self-interstitial defect in diamond is found to produce two low-intensity peaks in the Raman spectrum above the first-order peak, at about 1550 and 1930 $\mathrm{cm}^{-1}$ (at infinite defect dilution), which are assigned to welllocalized stretching atomic motions on the defect. The former mode is also IR active, produces a very intense peak (whose intensity increases as the defect concentration decreases), and most likely corresponds to the intense experimental IR feature reported at $1570 \mathrm{~cm}^{-1}$, thus corroborating the tentative assignation to the self-interstitial defect. A second very intense IR peak appears at about $500 \mathrm{~cm}^{-1}$, which corresponds to a "wagging" motion of the self-interstitial defect and exhibits a more collective, less localized character.

Present results highlight the reliability and effectiveness of state-of-the-art quantum-mechanical techniques based on the use of hybrid functionals in describing a variety of properties of defective solids.

Acknowledgments CMZW thanks Mexican CONACyT for financial support through project CB-178853.

\section{References}

1 S. J. Breuer and P. R. Briddon, Phys. Rev. B, 1995, 51, 6984 6994.

2 A. Mainwood, Diamond Relat. Mater., 1999, 8, 1560-1564.

3 R. Kalish, A. Reznik, S. Prawer, D. Saada and J. Adler, Phys. Status Solidi A, 1999, 174, 83-99.

4 G. Davies, B. Campbell, A. Mainwood, M. Newton, M. Watkins, H. Kanda and T. Anthony, Phys. Status Solidi A, 2001, 186, 187-198.

5 J. P. Goss, B. J. Coomer, R. Jones, C. J. Fall, P. R. Briddon and S. Öberg, Phys. Rev. B, 2003, 67, 165208.

6 J. Goss, R. Jones, T. Shaw, M. Rayson and P. Briddon, Phys. Status Solidi A, 2001, 186, 215-220.

7 G. Davies, H. Smith and H. Kanda, Phys. Rev. B, 2000, 62, 1528-1531.

8 R. Kalish, A. Reznik, K. Nugent and S. Prawer, Nucl. Instrum. Meth. Phys. Res. B, 1999, 148, 626-633.

9 D. Twitchen, D. Hunt, M. Newton, J. Baker, T. Anthony and W. Banholzer, Physica B: Condensed Matter, 1999, 273, 628631.

10 P. Lai, S. Prawer and C. Noble, Diamond Relat. Mater., 2002, 11, 1391-1396.

11 A. Moroño, S. G. de Vicente and E. Hodgson, Fusion Eng. Des., 2007, 82, 2563-2566.

12 H. Amekura and N. Kishimoto, J. Appl. Phys., 2008, 104, 63509.

13 S. Prawer, I. Rosenblum, J. O. Orwa and J. Adler, Chem. Phys. Lett., 2004, 390, 458-461.

14 D. Hyde-Volpe, B. Slepetz and M. Kertesz, J. Phys. Chem. C, 2010, 114, 9563-9567.

15 A. C. Ferrari and J. Robertson, Phys. Rev. B, 2000, 61, 1409514107.

16 A. C. Ferrari and J. Robertson, Phys. Rev. B, 2001, 64, 075414.

17 D. N. Jamieson, S. Prawer, K. W. Nugent and S. P. Dooley, Phys. Rev. B, 1995, 106, 641-645.

18 J. D. Hunn, S. P. Withrow, C. W. White and D. M. H. Jr., Phys. Rev. B, 1995, 52, 8106-8111.

19 S. Prawer, K. W. Nugent and D. N. Jamieson, Diamond Relat. Mater., 1998, 7, 106-110.

20 J. O. Orwa, K. W. Nugent, D. N. Jamieson and S. Prawer, Phys. Rev. B, 2000, 62, 5461-5472.

21 R. Brunetto, G. A. Baratta and G. Strazzulla, J. Appl. Phys., 2004, 96, 380-386.

22 P. Olivero, S. Rubanov, P. Reichart, B. C. Gibson, S. T. Huntington, J. R. Rabeau, A. D. Greentree, J. Salzman, D. Moore, D. N. Jamieson and S. Prawer, Diamond Relat. Mater., 2006, 15, 1614-1621.

23 A. A. Bergman, A. M. Zaitsev, M. Huang and A. Gorokhovsky, J. Lumin., 2009, 129, 1524-1526.

24 J. Goss, B. Coomer, R. Jones, T. Shaw, P. Briddon, M. Rayson and S. Öberg, Phys. Rev. B, 2001, 63, 195208.

25 C. A. Coulson and M. J. Kearsley, Proc. R. Soc. A, 1957, 241, 433-454. 
26 L. Maschio, B. Kirtman, R. Orlando and M. Rérat, J. Chem. Phys., 2012, 137, 204113.

27 L. Maschio, B. Kirtman, M. Rérat, R. Orlando and R. Dovesi, J. Chem. Phys., 2013, 139, 164102.

28 L. Maschio, B. Kirtman, M. Rérat, R. Orlando and R. Dovesi, J. Chem. Phys., 2013, 139, 167101.

29 C. Carteret, M. De La Pierre, M. Dossot, F. Pascale, A. Erba and R. Dovesi, J. Chem. Phys., 2013, 138, 014201.

30 L. Maschio, B. Kirtman, S. Salustro, C. M. Zicovich-Wilson, R. Orlando and R. Dovesi, J. Phys. Chem. A, 2013, 117, 11464-11471.

31 M. Prencipe, L. Maschio, B. Kirtman, S. Salustro, A. Erba and R. Dovesi, J. Raman Spectrosc., 2014, 45, 703-709.

32 J. Maul, A. Erba, I. M. G. Santos, J. R. Sambrano and R. Dovesi, J. Chem. Phys., 2015, 142, 014505.

33 R. Dovesi, R. Orlando, A. Erba, C. M. Zicovich-Wilson, B. Civalleri, S. Casassa, L. Maschio, M. Ferrabone, M. De La Pierre, Ph. D’Arco, Y. Noël, M. Causá, M. Rérat and B. Kirtman, Int. J. Quantum Chem., 2014, 114, 1287-1317.

34 L. Bjaalie, A. Janotti, K. Krishnaswamy and C. G. Van de Walle, Phys. Rev. B, 2016, 93, 115316.

35 M. Gerosa, C. Di Valentin, C. E. Bottani, G. Onida and G. Pacchioni, J. Chem. Phys., 2015, 143, 111103.

36 M. Gerosa, C. E. Bottani, L. Caramella, G. Onida, C. Di Valentin and G. Pacchioni, J. Chem. Phys., 2015, 143, 134702.

37 I. d. P. R. Moreira and R. Dovesi, Int. J. Quantum Chem., 2004, 99, 805-823.

38 D. Munoz, N. M. Harrison and F. Illas, Phys. Rev. B, 2004, 69, 085115 1-9.

39 J. C. Wojdeł, I. de P. R. Moreira, S. T. Bromley and F. Illas, J. Chem. Phys., 2008, 128, 044713 1-9.

40 J. Baima, A. Zelferino, P. Olivero, A. Erba and R. Dovesi, Phys. Chem. Chem. Phys., 2016, 18, 1961-1968.

41 A. Zelferino, S. Salustro, J. Baima, V. Lacivita, R. Orlando and R. Dovesi, Theor. Chem. Acc., 2016, 135, 1-11.

42 A. D. Becke, J. Chem. Phys., 1993, 98, 5648-5652.

43 C. Lee, W. Yang and R. G. Parr, Phys. Rev. B, 1988, 37, 785789.

44 P. Dirac, Proc. R. Soc. A, 1930, 126, 360-365.
45 J. P. Perdew, K. Burke and M. Ernzerhof, Phys. Rev. Lett., 1996, 77, 3865-3868.

46 C. Adamo and V. Barone, Chem. Phys. Lett., 1998, 298, 113 119.

47 A. V. Krukau, O. A. Vydrov, A. F. Izmaylov and G. E. Scuseria, J. Chem. Phys., 2006, 125, 224106-224106.

48 J. S. Binkley, J. A. Pople and W. J. Hehre, J. Am. Chem. Soc., 1980, 102, 939-947.

49 M. M. Francl, W. J. Petro, W. J. Hehre, J. S. Binkley, M. S. Gordon, D. J. DeFrees and J. A. Pople, J. Chem. Phys., 1982, 77, 3654-3665.

50 F. Pascale, C. M. Zicovich-Wilson, F. L. Gejo, B. Civalleri, R. Orlando and R. Dovesi, J. Comput. Chem., 2004, 25, 888897.

51 C. Zicovich-Wilson, F. Pascale, C. Roetti, V. Saunders, R. Orlando and R. Dovesi, J. Comput. Chem., 2004, 25, 1873-1881.

52 A. Erba, M. Ferrabone, R. Orlando and R. Dovesi, J. Comput. Chem., 2013, 34, 346-354.

53 J. Baima, M. Ferrabone, R. Orlando, A. Erba and R. Dovesi, Phys. Chem. Minerals, 2016, 43, 137-149.

54 M. Ferrero, M. Rérat, R. Orlando and R. Dovesi, J. Comput. Chem., 2008, 29, 1450-1459.

55 M. Ferrero, M. Rérat, R. Orlando and R. Dovesi, J. Chem. Phys., 2008, 128, 014110.

56 G. M. Barrow, Introduction to molecular spectroscopy, McGrawHill, New York, 1962, p. 70.

57 B. A. Hess, L. J. Schaad, P. Carsky and R. Zahradnik, Chem. Rev., 1986, 86, 709-730.

58 S. B. Zhang and J. E. Northrup, Phys. Rev. Lett., 1991, 67, 2339-2342.

59 C. G. V. de Walle, D. B. Laks, G. F. Neumark and S. T. Pantelides, Phys. Rev. B, 1993, 47, 9425-9434.

60 C. Freysoldth, B. Grabowski, T. Hickel, J. Neugebauer, G. Kresse, A. Janotti and C. G. V. de Walle, Rev. Mod. Phys., 2014, 86, 253-305.

61 Graphical animations of the vibration modes of the self-interstitial defect in diamond available at: http://www.crystal.unito.it/Jmolvib/index.html?cartesian $=$ interstitial.xyz . 